\title{
Germanica
}

\section{Comment Nietzsche comprit Platon}

Wie Nietzsche Plato's Philosophie verstand

\section{Pierre Trotignon}

\section{CpenEdition}

Journals

Édition électronique

URL : http://journals.openedition.org/germanica/2426

DOI : 10.4000/germanica.2426

ISSN : 2107-0784

Éditeur

Université de Lille

\section{Édition imprimée}

Date de publication : 31 décembre 1990

Pagination : 11-28

ISBN : 9782913857025

ISSN : 0984-2632

Référence électronique

Pierre Trotignon, «Comment Nietzsche comprit Platon », Germanica [En ligne], 8 | 1990, mis en ligne le 25 novembre 2014, consulté le 06 octobre 2020. URL : http://journals.openedition.org/germanica/

2426 ; DOI : https://doi.org/10.4000/germanica.2426

Ce document a été généré automatiquement le 6 octobre 2020.

(c) Tous droits réservés 


\title{
Comment Nietzsche comprit Platon
}

\author{
Wie Nietzsche Plato's Philosophie verstand
}

\section{Pierre Trotignon}

1 L'antiplatonisme de Nietzsche est bien connu et bien établi. On peut lire sous la plume de Nietzsche des déclarations telles que celles-ci, datée de 1870: «Ma philosophie est un platonisme renversé : plus on s'éloigne de l'être vrai, plus cela est purement beau et meilleur. Le but, c'est : la vie dans l'apparence $»^{1}$. On ne saurait être à la fois plus net ni plus provocant. Après quoi, il serait permis de se demander si une telle assertion nous laisse l'espoir d'attendre de la part de son auteur des analyses du platonisme qui soient autre chose qu'une fin de non-recevoir. Et cependant la manière dont Nietzsche présente Platon est l'une de ses analyses les plus étonnantes, et, parfois, des plus instructives.

2 Nietzsche, qui avait fait de solides études classiques et philologiques, connaissait Platon directement. Dans le texte de 1864, il a donc 20 ans, intitulé Ma vie, il écrit : «Je pense avec un souvenir très agréable à mes premières impressions de Sophocle, d'Eschyle, de Platon... du Banquet $»^{2}$. Et, de fait, on possède encore une dissertation du jeune Nietzsche sur le Banquet de Platon ${ }^{3}$. Rien n'y annonce la future philosophie de Nietzsche, mais on remarquera, toutefois, que l'opposition de Socrate et d'Alcibiade y est présentée comme signe de la double nature d'Erôs, de son lien avec le divin et l'humain, le sensible et l'intelligible. Discrètement on entend sonner les premières notes du grand combat. Mais qu'on lise les textes de Nietzsche devenu maittre de sa pensée, on y voit que l'aversion pour Platon a remplacé cette timide analyse. Voici un texte de 1884: «... ma vieille aversion pour Platon, l'anti-antique, l'âme moderne existait déjà »", car l'affaiblissement de la vie dans l'âme moderne a sa source dans le reflux socratique devant la pensée artiste de ses prédécesseurs. Nietzsche est, en effet, l'un des premiers auteurs à jouer de cette opposition entre les présocratiques et les post-socratiques. Il discerne chez Platon les symptômes de la décadence qu'il apercevait dans le sentiment rousseauiste ou la volonté schopenhauerienne qui, poursuivant le mouvement inauguré par Pascal, s'achève en pitié, en renoncement, en négation, en nihilisme. 
3 Mais, tout comme il pousse à bout le nihilisme entravé de Schopenhauer, en un double jeu de sympathie et de réfutation, il adopte à l'égard de Platon une attitude double, ambiguë. Et, d'abord, parce qu'il conçoit Platon comme étant lui-même double : une volonté forte et dominatrice qui n'a malheureusement pas d'autre objet à se soumettre que soi-même. Ainsi il écrit en 1880 :

Aux époques de dissolution, les grandes personnalités morales sont celles qui savent se tenir en bride. Signe d'orgueil, car ce sont des tempéraments dominateurs (Heraclite, Platon) dans un monde changé, où ils n'ont plus à dominer qu'euxmêmes ${ }^{5}$.

Donc ne pas simplifier: Platon n'est pas un moraliste vulgaire, bien que ce soit l'interprétation morale de l'être qui soit le fil conducteur de sa philosophie. Ce qui explique un mélange de refus et de séduction :

Les esprits dogmatiques comme Dante et Platon, écrit Nietzsche, sont ceux dont je me sens le plus éloigné et peut-être de ce fait ceux qui ont pour moi le plus de charme; ceux qui habitent dans des demeures bien charpentées et, à ce qu'il semble, solides, de la connaissance ${ }^{6}$.

5 Mais Nietzsche maintient qu'il faut encore plus de vigueur pour vivre dans un système inachevé. Le chemin de la sagesse comporte une longue pédagogie : vénérer toutes les valeurs, les faire s'affronter en nous, puis briser, partir au désert et s'y faire enfin pure affirmation dans un monde qui ne comporte en soi ni sens ni finalité. Et Nietzsche se demande en 1884 : «Platon y aurait-il réussi ? $»^{7}$. Peut-être, car il fait partie de ces hommes qui sont plus puissants que l'artiste qui est en eux, de sorte que Platon valait mieux que sa philosophie : "s'il est vrai que Platon ressemblait à ce buste qui est à Naples, c'est la meilleure réfutation de tout le christianisme" (texte de 1884) ${ }^{8}$. Autrement dit, Platon est un aristocrate séduit par Socrate le plébéien : Platon aurait naïvement pris à la lettre l'identification socratique du logos et du bien, et cette "naiserie» aurait été vulgarisée par le christianisme, nous tiendrons là le motif de l'asservissement de l'esprit européen. L'explication qu'en donne Nietzsche est curieuse : Platon a tort de poser et définir le Bien suprême, puisqu'il représente luimême le bien suprême, c'est-à-dire l'homme supérieur. Le platonisme reposerait ainsi sur la honte d'avouer sa puissance et l'universalisme rationnel de Socrate lui offrait une porte de sortie, par projection et complicité avec la volonté faible. Nietzsche va jusqu'à supposer que Platon était trop intelligent pour croire à sa propre doctrine :

Platon n'était certes pas borné au point de croire, comme il l'enseignait, que les concepts sont fixes et éternels, mais il voulait qu'on le crût ${ }^{9}$.

Non par cynisme, mais par faiblesse, parce qu'il avait bridé l'artiste en lui.

7 La morale contre l'art. Tel est le noyau de la représentation que Nietzsche se fait de la philosophie de Platon: "Depuis Platon, écrit-il en 1883, la philosophie est sous la domination de la morale ${ }^{10}$, qui, en imaginant une distinction et un conflit de deux principes, permet à la volonté affaiblie de s'illusionner sur le sens de sa docilité et, en même temps, d'exiger des volontés créatrices qu'elles se ploient à la routine faussement rationnelle. Le " tu dois » universel serait un "je veux » brisé et dissimulé, revenant en écho du ciel où il a été projeté. Ainsi Nietzsche subordonne toute la théorie platonicienne du savoir et toute son ontologie des Idées éternelles à cette intention morale. La dialectique repose sur un préjugé moral, qui est celui de la véracité d'un Dieu bon, garant de l'éternité des valeurs, assurant ainsi une confiance de mauvaise foi en une existence précaire. Nietzsche interprète ainsi le lien entre dialectique, maïeutique, réminiscence et chute de l'âme dans le monde. Mais la volonté artiste, qui 
s'est laissée encanailler par Socrate, va commettre une lourde erreur : comme elle ne peut renoncer à sa puissance, qui pourtant ne lui apparait plus que comme un universel externe, elle nourrira l'illusion de faire descendre sur terre la Cité Idéale où serait sauvegardée l'aristocratie, par le biais de la royauté philosophique. Nietzsche écrit en 1884 : «Platon a voulu créer une caste dont l'existence soit liée à la fixité définitive des jugements moraux, comme à son intérêt vital, - la classe des bons et des justes " ${ }^{11}$. D'où la République de Platon. Mais elle recèle une contradiction essentielle: elle veut protéger les meilleurs par le mensonge sacré (poésie d'Etat) mais se veut en même temps une pédagogie pour améliorer tout homme. Nietzsche écrit en 1884 :

Naïveté de Platon et du christianisme : ils croyaient savoir ce qu'est le «bien». Ils avaient deviné l'homme du troupeau, non l'artiste créateur. Platon déjà a inventé le «Sauveur » qui descend chez les souffrants et les méchants. Il n'aperçoit pas la raison ni la nécessité du mal ${ }^{12}$.

Le paganisme reconnaissait le mal, le platonisme aurait empoisonné cette innocence naïve, et sa pudeur aveugle quant à la puissance des instincts a entrainé la destruction du paganisme. Il aurait ainsi inauguré le règne du fanatisme moral, et c'est pourquoi Nietzsche dit de Platon qu'il est une " caricature » : parce que la " lutte contre le mal », rend, à son corps défendant, celui qui s'y jette "mauvais ", sans aucune compensation, car ce mal réactif ne peut être en lui cause d'une création, de sorte que, dit Nietzsche " on peu en déduire infailliblement le jugement que le fanatique de la morale prononcera sur tout ce qui forme les cimes de l'évolution humaine : un non plus ultra de corruption. Songez au jugement de Savonarole sur Florence, de Platon sur l'Athènes de Périclès, de Luther sur Rome, de Rousseau sur la société voltairienne, des Allemands sur Goethe $»^{13}$. Ce primat de l'interprétation morale de l'être a pour conséquence un renversement de l'évaluation ontologique, qui est le propre de l'art. La morale ne s'oppose pas à l'art, elle en inverse le système de mesure. L'artiste préfère l'apparence au « réel », l'artiste pense que moins une chose est réelle, plus elle a de valeur. Or, dit Nietzsche, «c'est du platonisme ", mais Platon a eu la hardiesse inverse, il mesurait le degré de réalité, la positivité, la vérité d'après le degré de valeur et disait : " plus c'est une idée, plus cela est ». Il retournait la notion de réalité et disait : ce que vous tenez pour vrai est une erreur... $»^{14}$. Précisons bien ici le vocabulaire: Platon, en artiste, préfère l'apparence à l'être, mais en moraliste il confère à cette apparence les qualités qui, moralement parlant, définissent la réalité, la positivité, la vérité, la bonté. Nietzsche discerne ici une calomnie contre la vie, un dénigrement de la réalité.

9 Nous pouvons maintenant suivre plus en détail le développement de la critique nietzschéenne de Platon. La distinction entre l'idée et l'idole aurait pour fondement un trait du tempérament grec, l'opposition entre la puissance de Dionysos et l'individu qui n'est que le masque et le prête-nom, déclare Nietzsche au \$10 de L'Origine de la tragédie ${ }^{15}$. Ce qui est important, car le platonisme serait ainsi l'inverse de la doctrine pessimiste des Mystères, la volonté d'être heureux tout en demandant ce bonheur pour une individualité éternelle. La prédominance du nous sur la puissance de la vie est ainsi l'essence du socratisme, chez Platon comme chez Euripide. Le $\$ 12$ de L'Origine de la tragédie nous dit :

Euripide entreprit comme Platon de montrer au monde ce qu'est le contraire d'un poète déraisonnable ${ }^{16}$,

c'est-à-dire de mettre la conscience à la racine du beau et du bien. Or la tragédie dionysiaque est irrationnelle, et Socrate, - Nietzsche fait allusion ici au début du Phédon -, lui préférait le prosaïsme des fables d'Esope. Le privilège de l'utilitaire lui fait 
suspecter le plaisir de la représentation tragique. Platon, selon un fragment de l' Anthologie palatine, aurait détruit des tragédies de jeunesse et renoncé à l'art. Mais Platon ne peut rester, au fond de lui-même, qu'artiste, et la violence faite à cet art irrépressible engendre le genre du dialogue, où la poésie devient la servante de la philosophie, l'art dialectique d'argumenter et de donner des raisons de l'existence. Apollon ici écrase Dionysos. L'optimisme moral anéantit la tragédie, avec toutefois un remords, une nostalgie et le sentiment d'une perte. Le \$14 de la Naissance de la tragédie expose admirablement cette genèse du platonisme à partir de la tragédie dionysiaque ${ }^{17}$.

11 L'ouvrage de Platon qui demeure au centre de cette analyse est évidemment la République. L'état idéal de Platon serait la solution aux rapports de l'existence collective et du génie, du moins tel que Platon peut le vivre en un drame intérieur. La figure du tyran, qui est l'artiste passé à travers la morale et désirant s'en libérer, fascine Platon, et Nietzsche n'a garde d'oublier ses relations avec Denys de Syracuse ${ }^{18}$. Cette image du tyran hantera Nietzsche toute sa vie, sa racine est la méditation sur la contradiction entre l'art et la métaphysique dans l'œuvre de Platon. Car Platon demeure lié, même dans la polémique de Nietzsche dans Homers Wettkampf, avec l'art des sophistes et des dramaturges, au point qu'il invente comme eux des mythes et des drames. À l'inverse de Bergson, qui verra Socrate dénaturé par Platon, Nietzsche pense que Socrate a dévoyé Platon en lui injectant le virus dialectique et moral. Ainsi ${ }^{19}$, dans Humain, trop humain au \$261:

Ce n'est pas une question oiseuse de se demander si Platon, resté libre du charme socratique, n'aurait pas trouvé un type plus élevé encore d'homme philosophique, perdu pour nous à jamais.

Ainsi s'esquisse l'ensemble de la perspective nietzschéenne sur Platon : il est considéré comme l'exemple idéal de l'artiste qui renonce à l'art au profit de la vérité, l'exemple de la force créatrice qui se laisse dominer par le vertige du bien. L'attitude de Nietzsche à l'égard de Wagner éclaire assez bien l'idée qu'il se faisait de Platon.

13 La question centrale de la République, à savoir la question d'une pédagogie de la raison dans le genre humain, retient particulièrement l'attention de Nietzsche, mais surtout parce que Nietzsche est frappé de la stérilité et des dangers de la fausse culture dont il dénonce les méfaits en Allemagne. Et tout de même que l'état platonicien reposait sur un « mensonge de sécurité » (Notlüge), qui est dans la République (III, 414-415), la fiction des autochtones et de la fixité de l'ordre social, de même la culture allemande repose sur le mensonge de la formation purement historique. Et Nietzsche poursuit la comparaison, dans la seconde des Considérations intempestives, au $\$ 10^{20}$, en disant que ce système s'écroulera comme la « république » de Platon, parce que dans les deux cas la vie a été niée au profit d'une catégorie ontologique vide. Le malheur veut que «Platon ait eu dans l'histoire une singulière malchance: chaque fois que l'on a vu naître une construction conforme à ses principes, au moins dans les grandes lignes, ce n'était, à y regarder de près, qu'un enfant de lutin, substitué au sien, un affreux avorton $»^{21}$. Mais il est des mésaventures dont on porte parfois la responsabilité. Car si l'Etat ne peut sincèrement vouloir que la philosophie soit libre, comment Platon a-t-il pu croire que son Etat échapperait à cette loi ? D’ailleurs, que dit-il, en République, II, 378-379 : « Pour le moment, ni toi, ni moi ne sommes des poètes, mais des fondateurs d'État». Et Nietzsche dit encore :

En proie à ce mélange de puissante volupté et de crainte, il faut alors être un Platon,

à tout le moins, pour oser, comme il l'a fait, dire au poète tragique: "nous

honorerons dans notre république, comme un être sacré et miraculeux, l'homme à 
qui sa sagesse permettrait de devenir tout au monde et d'imiter toutes choses, nous oindrons sa tête de parfums et nous le couronnerons de bandelettes, mais nous tâcherons de le persuader d'aller se fixer dans un autre État $"^{22}$. tyran, " on risque fort, dit Nietzsche dans le Crépuscule des idoles ${ }^{27}$ de voir autre chose exercer de fait la tyrannie. En effet, ce besoin de la lumière sans cette allumée trahit la crainte de la nuit, l'impossibilité de la supporter et donc la forme la plus subtile de domination de l'obscur. La peur de s'abaisser en se fiant à l'instinct trahit la peur panique d'une vie en détresse. Identifier raison, vertu et bonheur, c'est se proposer de faire le bonheur de l'homme en l'améliorant et en usant de tous les moyens, de sorte que "pour faire de la morale ", dit Nietzsche dans le Crépuscule des idoles ${ }^{28}$, «il faut absolument aussi la volonté contraire [...] Ni Manou, ni Platon, ni Confucius, ni les maîtres juifs et chrétiens n'ont jamais douté de leur droit au mensonge [...]. Si l'on voulait s'exprimer en formule, on pourrait dire : tous les moyens par lesquels jusqu'à présent l'humanité devait être rendue plus morale étaient foncièrement immoraux ».

Ainsi Platon serait le type même de l'esprit qui se mystifie et se corrompt pour devenir meilleur, illusoirement meilleur, par un amour de la vérité qui cache l'inauthencité de la vie faible.

21 À la racine de cette volonté du vrai et du bien absolu, il y a la croyance en l'idéal. Mais sur ce point la position de Platon est ambiguë, puisqu'il est séduit par Socrate tout en conservant une veine esthétique. Nietzsche voit comme un signe de cette ambiguïté 
dans le lien qui unit la philosophie de Platon à l'âge auquel il découvrit sa voie propre. Il écrit dans Le Voyageur et son ombre, tome II, \$271 ${ }^{29}$ :

L'âge de la vie auquel un philosophe découvrit sa doctrine se reconnaît dans son œuvre [...] c'est ainsi que la philosophie de Platon rappelle le milieu de la trentaine, époque où un courant froid et un courant chaud se rencontrent généralement avec impétuosité, soulevant de la poussière et de petits nuages ténus mais faisant naître, dans des circonstances favorables, lorsque le soleil donne, un arc-en-ciel enchanteur.

Mais il peut arriver que le soleil ne brille plus, et il est bien certain que la mort de Socrate fut pour Platon une injustice qui requérait une interprétation du sens du monde. Platon ne put résorber la mort de Socrate par la conjuration de son œuvre, qui dut, à la fin, faire elle aussi, disparaître Socrate, de sorte que la fin de l'œuvre de Platon, où Socrate n'est plus le maître qui enseigne, témoigne d'une reprise de la doctrine quelque peu déçue et amère :

Au lieu d'examiner à nouveau les résultats, dit Nietzsche dans Aurore, $\$ 542^{30}$, et de recommencer à les semer, on a besoin de les apprêter à un goût nouveau, pour se les rendre supportables et leur enlever leur sécheresse, leur froideur et leur manque de saveur. C'est ce qui fait que le vieux penseur s'élève en apparence audessus de l'œuvre de sa vie, mais qu'en réalité il la gâte par l'exaltation, les douceurs, les épices, la brume poétique et les lumières mystiques qu'il y mêle. C'est ce qui finit pas arriver à Platon....

L'enthousiasme dialectique, l'allégresse du dialogue font place à la sécheresse logique. Platon, dont Nietzsche dit qu'il n'aurait pu exister sans l'art tragique qui le précède ${ }^{31}$, transmue et transpose le tragique en science logique : «Métastase de l'instinct tragique artiste dans la science. Socrate et Platon: la culture $\aleph^{32}$. On comprend comment le « renversement des valeurs ", l'Umwertung aller Werten, de Nietzsche prend le contrepied de la révolution socratique et platonicienne.

Cette révolution platonicienne, Nietzsche la comprend non comme une simple invention d'idées mais comme un changement radical de l'homme, une évaluation de la vie dans sa totalité. C'est ainsi que le $\$ 408$ de la première partie du Voyageur et son ombre $e^{33}$ place Platon parmi les esprits fondamentaux, apparenté à Rousseau, opposé à Goethe et à Spinoza. La parenté avec Rousseau est évidemment lié à la morale et à la pédagogie socratiques. Car Platon reste profondément aristocrate et il n'aurait pas suivi Rousseau sur le chemin de la pitié et de la sensiblerie :

Que l'on se souvienne donc de la façon dont Platon - qui n'était pas un des philosophes les plus inhumains - parle du Philoctète de la scène tragique » (Aurore, $\$ 157)^{34}$.

Allusion évidente à la critique platonicienne de l'art épique et tragique. Pour Nietzsche, Platon sur ce point est ambigu et son ambiguïté contraste avec la solidité massive des philosophes présocratiques. Cette ambiguïté sera le terrain de la mauvaise conscience et de l'intériorisation de la mauvaise conscience par l'homme qui se cache ses conflits avec le destin. On passe de l'héroïsme à la défaite intérieure. Le platonisme est hybride. Nietzsche écrit en 1875 :

Platon lui-même est le premier grand hybride, tant dans sa philosophie que dans sa personne. Sa doctrine des Idées comporte des éléments socratiques, pythagoriciens et héraclitéens, c'est pourquoi elle n'est pas un phénomène de type pur. Comme homme aussi, Platon réunit en lui la réserve royale et la sérénité auguste d'Héraclite, la compréhension mélancolique du législateur Pythagore et la dialectique du connaisseur d'âmes Socrate. Tous les philosophes ultérieurs sont de tels hybrides ${ }^{35}$. 
Il est clair que pour Nietzsche l'élément étranger dans cette hybridation, comme il le dira clairement au $\$ 190$ de Par-delà le bien et le mal ${ }^{36}$, c'est le socratisme, qui répugnait, au fond, au caractère aristocratique de Platon.

Qu'est le socratisme? Pour Nietzsche, il tient tout entier dans cette affirmation plébéienne que le mal découle d'une erreur de jugement et qu'il suffit de rectifier l'esprit pour voir comment le bien peut seul être utile et rendre heureux. Que fit donc Platon pour composer ce principe socratique et les autres ingrédients de sa philosophie ? Voici la réponse de Nietzsche, au $\$ 190$ de Par-delà le bien et le mal $^{37}$ : «Platon a tout fait pour interpréter à sa guise le précepte de son maître (hineinzuinterpretieren) », pour y introduire un peu de délicatesse et de noblesse, pour s'y introduire lui-même d'abord, lui le plus audacieux de tous les interprètes (« er, der verwegenste aller Interpreten »)..., et cette interprétation est à son tour interprétée par Nietzsche comme une série de variations, prêtant à Socrate tous les masques de la figure chatoyante et versatile de Platon. Et Nietzsche ajoute : «Pour le dire en termes plaisants, et en termes de plaisanterie homérique de surcroît, qu'est-ce que le Socrate de Platon, sinon : "prosthe Platon, opisthen Platon, messe te Khimaîra ", Platon par devant, Platon par derrière et la Chimère au milieu $»^{38}$. Or il n'est pas sans intérêt de noter que Nietzsche ici pastiche un pastiche, puisqu'il utilise un vers d'Homère ${ }^{39}$ qui avait déjà servi à caractériser plaisamment Arcésilas, dont Ariston dit qu'il était Platon par devant, Pyrrhon par derrière et Diodore au milieu. Car on rejoint ici une longue tradition, celle de l'interprétation sceptique de Platon. Platon serait un sceptique et le platonisme un hybride de scepticisme et de socratisme.

Le produit de cette incertitude de la volonté et de la certitude morale serait l'érotique platonicienne Nietzsche dit, dans le Crépuscule des idoles, au \$23 des Flâneries inactuelles:

Il faudrait définir la philosophie, telle que la pratiquait Platon, comme une sorte de lice érotique, continuant et approfondissant la vieille gymnastique agonale et toutes les conditions qui précédaient [...] qu'est-il résulté, en dernier lieu, de cet érotisme philosophique de Platon? Une nouvelle forme d'art de l'âgon grec, la dialectique $^{40}$.

29 À vrai dire cette sublimation sexuelle (Charmide, Banquet, Phèdre) est à l'honneur de Platon. Mais l'élément socratique infléchit cette érotique en un amour du vrai qui suppose la persécution de soi, le déni du sensible et le mépris pour la poésie. Dans le $\S 91 \mathrm{du}$ Gai Savoir, Nietzsche dit que les autobiographies sont des menteries par despostisme envers soi-même pour se plier à un modèle, et il ajoute, curieusement :

Je n'accorderais pas plus de créance à une autobiographie de Platon, tout aussi peu qu'à celle de Rousseau ou à la Cita nuova de Dante ${ }^{41}$.

C'est laisser entendre que toute l'œuvre de Platon est cette confession d'une lutte intérieure entre l'art et la morale.

C'est donc par la séduction de la morale, cette "Circé des philosophes", comme dit Nietzsche au $\$ 3$ d'Aurore, que Socrate a infecté Platon. Cette séduction a un sens très clair : la volonté faible, qui ne veut plus se risquer et se perdre dans l'affrontement tragique où elle se dépasse, ne pouvant pas vaincre la puissance qui la gêne et l'humilie, va tenter de lui faire adopter, comme mesure universelle du bien, ce qu'elle-même est capable de supporter, et, par là-même, de disqualifier, sous le nom de mal, les énergies puissantes et créatrices. Ce soupçon jeté sur la force a pour effet une sur-évaluation de l'intériorité scrupuleuse, un primat de l'intention sur l'effectivité, autrement dit la théorie du salut par la foi : «Cette doctrine du salut par la foi, écrit Nietzsche dans le 
\$22 d'Aurore, n'est tout bonnement pas vraie, mais elle a l'air si séduisante qu'elle a déjà ébloui des intelligences bien autres que celle de Luther (je veux dire celles de Socrate et de Platon) $»^{42}$. Il est vrai que Socrate se faisait le héraut de la raison, non de la foi, mais précisons la pensée de Nietzsche. La foi que critique Socrate est la confiance de l'instinct créateur en lui-même, la raison qu'il invoque est la confiance de la pensée en un autre transcendant, et, selon Nietzsche, (cf. Par-delà le bien et le mal ; \$191), Socrate savait fort bien que l'instinct est la puissance motrice réelle, mais, ajoute-t-il, «Platon, plus innocent en ces matières, dépourvu de toute astuce plébéienne, voulut de toutes ses forces - et elles dépassaient de beaucoup celles de ses prédécesseurs, se démontrer à lui-même que la raison et l'instinct tendent au même but, au bien, à Dieu $»^{43}$. Pour Nietzsche cette victoire de la foi, dans le platonisme, est celle du troupeau sur l'élite, car si les forts aspirent à se séparer et visent la tyrannie créative, les faibles veulent s'agglutiner - le conflit de Platon en est la preuve, et Platon le savait, nous dit le $\$ 18$ de la troisième dissertation de La généalogie de la morale ${ }^{44}$.

Pendant l'hiver 1872, dans les Considérations sur le conflit de l'art et de la connaissance, Nietzsche a dit que la singularité de Platon est son « enthousiasme pour la dialectique, c'est-à-dire pour le sang-froid conscient $»^{45}$. Ce sang-froid conscient (l'imagine-t-on chez un artiste ?) est la séparation méthodique d'avec le sensible, l'ascension ascétique et dialectique vers le monde supérieur des Idées. "De là », dit Nietzsche dans le $\$ 23 \mathrm{~d}$ 'Aurore, "l'admiration de Platon pour la dialectique ${ }^{46}$, de là sa foi enthousiaste en les rapports nécessaires de "celle-ci avec l'homme bon, délivré de sens» (ibidem). Ou encore, dans le même ouvrage, au $\$ 474$ : «la dialectique est le seul chemin pour parvenir à l'être divin, pour parvenir derrière le voile de l'apparence - c'est ce que Platon prétendait $»^{47}$.

Mais pourquoi ? Parce que dans ce renoncement et cet effort pour « dompter le monde et l'interpréter il y avait une jouissance» (Par-delà le bien et le mal, § 14) ${ }^{48}$. Car connaître aussi procure de la jouissance. Voir, par exemple, le $\$ 550$ de Aurore : «Platon et Aristote s'entendirent sur ce qui constitue le bonheur suprême, non seulement pour eux et pour les hommes, mais le bonheur en soi-même, pour les Dieux et les dernières béatitudes : ils le trouvèrent dans la connaissance, dans l'autorité d'une raison exercée à trouver et s'inventer... $\star^{49}$. Mais il demeure bien figuratif et instable, ce plaisir de voir l'essence cachée, et l'ascétisme va prétendre que je dois avoir foi dans l'intelligible sans plaisir aucun. Le platonisme devient ainsi la préparation au christianisme et le christianisme ne sera qu'un platonisme pour le peuple ${ }^{50}$.

Il y eut, dit Nietzsche une "tentative de Platon pour devenir un rédempteur ${ }^{51}$. Car si je dois atteindre le vrai sans plaisir, qu'au moins ma souffrance serve à améliorer ceux qui sont encore dans l'ignorance. D'où cette idée, exprimée dès 1873, que «Socrate voulait sa mort » et admirablement reprise dans le $\$ 340$ du Gai Savoir, où la mort voulue guérit de la maladie de la vie.

Réformer l'homme en lui imposant une morale et en mourant soi-même pour cette morale, tel est le dessein, que le christianisme reprit avec succès. "Quelque hasard de plus, quelques autres hasards en moins » écrit Nietzsche dans le $\$ 496$ d'Aurore, «et le monde aurait assisté à la platonisation du midi européen, et en admettant que cet état de choses durât encore, il serait probable que nous adorerions aujourd'hui en Platon le principe bon $»^{52}$. Platon échoua parce que, nous dit le Gai Savoir au $\$ 149$, le monde grec était trop civilisé pour admettre l'uniformité et qu'il répugnait aux instincts grégaires ${ }^{53}$. L'« immoralité » grecque, que les théologiens chrétiens dénonceront, était le signe de la 
santé métaphysique des Grecs. À la force des types de sagesse, divers, multicolores des présocratiques, s'oppose la fausse modestie de Platon, qui est, en fait, comme Nietzsche le souligne dans le Gai Savoir (\$351) $)^{54}$ un monstrueux orgueil, que le moralisme a intériorisé en une lutte ascétique contre soi-même. Ce platonisme moral va calomnier l'homme et le monde qui le font souffrir, attitude qui l'oppose à Thucydide (Aurore, $\$ 168)^{55}$ et encore également à Homère (Généalogie de la morale, III, \$25) ${ }^{56}$. C'est cette calomnie et ce désespoir caché qui vont le pousser à «se réfugier au pays des Idées éternelles, dans l'atelier de l'artiste de l'univers, pour y repaître son regard parmi les prototypes immaculés et infrangibles des choses, mais dans la paix cadavérique et figée du concept le plus froid, du moins expressif de tous, de l'être " ${ }^{57}$. La théorie de "l'ontôs on » est ainsi comprise comme un refus du devenir ${ }^{58}$. L'interprétation morale de l'être suprasensible en ce que Nietzsche nomme l'idéalisme. Dans le \$2 du texte Ce que je dois aux Anciens dans le Crépuscule des idoles ${ }^{59}$, Nietzsche refuse d'admettre et d'admirer l'art de Platon, qui est une décadence du style, un baroque hybride, preuve de ce qu'il a dévié de l'instinct grec : « je le trouve si imprégné de morale, si chrétien avant la lettre, il donne déjà l'idée du bien comme idée supérieure - que je suis tenté d'employer à l'égard de tout le phénomène Platon plutôt que toute autre épithète celle de " haute fumisterie » (höherer Schwindel) ou, si l'on préfère, d'idéalisme ». "Idéalisme » signifie donc clairement : lâcheté devant le réel et fuite dans la brume. Chez Platon, il est la "prudence d'une santé dangereuse » qui a honte de soi à cause de Socrate (Gai Savoir, $\S 372)^{60}$, qui doit abolir le perspectivisme et en souffre, en est «malade » dit Par-delà le bien et le mal dans sa préface. La foi dans le savoir est une piété ${ }^{61}$, la vérité à tout prix une religion ${ }^{62}$. C'est elle qui impose la distinction absolue et l'antinomie entre l'âme et le corps, qui, écrit Nietzsche en 1875, "pèse, surtout depuis Platon, comme une malédiction sur la philosophie " ${ }^{63}$. Cet idéalisme est le signe d'une décadence: "J'ai reconnu en Socrate et en Platon des symptômes de décadence, des instruments de décomposition, des antigrecs $»^{64}$ (Crépuscule des idoles, le Problème de Socrate, \$2). "Antigrec »: en ce sens que l'idée du bien comme principe suprême de l'être est à l'origine de deux chimères. La première est la croyance en un ordre unique, simple, moral et finalisé du monde (et Nietzsche soulignait en $1875^{65}$ que Platon avait critiqué Anaxagore, dans le Phédon, parce qu'il n'était pas allé au bout de sa théorie de l'intellect). La seconde chimère, qui découle de la première, est la croyance en une donation du sens au monde sensible et à la vie de l'homme par la pensée. La première chimère platonicienne conduit à l'optimisme leibnizien, la seconde à la liberté intelligible de Kant (Crépuscule des idoles : les quatre grandes erreurs, \$8) ${ }^{66}$.

Donc, sous couvert de vérité absolue le platonisme serait un mensonge. Dans l'Antichrist, au §55, Nietzsche écrit : « Le saint-mensonge - commun à Confucius, au livre de Manou, à Mahomet et à l'Église chrétienne, ce mensonge se retrouve chez Platon. La vérité est là, cela signifie partout : le prêtre ment $"{ }^{67}$.

Tel est, sommairement exposé, le Platon de Nietzsche. Initiateur de l'idéalisme, de la croyance en un monde intelligible, stable et pur, régi par le principe du bien et qui servirait à la volonté faible de compensation à la souffrance du devenir, à la souffrance et au chaos insondable de la réalité. Mais Platon n'est pas absolument identique au platonisme: on discerne en lui le conflit du socratisme et de l'art tragique, il est hybride, il persiste en lui de l'énergie grecque et un sens esthétique. Lisons ce que Nietzsche écrit à la fin du \$28 de Par-delà le bien et le mal.

Je ne sais rien qui m'ait fait plus rêver sur la nature énigmatique de Platon que ce petit fait qui nous a été si heureusement transmis : au chevet de son lit de mort, on 
ne trouva ni «bible» ni écrit égyptien, pythagoricien ou platonicien, mais un exemplaire d'Aristophane. Comment un Platon aurait-il pu supporter la vie - cette vie grecque à laquelle il disait non - sans Aristophane ?68

D’Aristophane comme de Pétrone, Nietzsche dit qu'ils sont intraduisibles en allemand. Leur légèreté, leur raillerie, leur manière de dire, en se jouant, des choses lourdes à penser et terribles à voir ${ }^{69}$, - comment les Philistins pourraient-ils les apprécier convenablement? Car le Platon de Nietzsche est lu par un regard qui ne peut se détacher de l'Allemagne, du danger qui la menace et qu'elle fait peser sur le monde. Platon, lui aussi, fut la borne de deux millénaires, et le platonisme finit par mettre la main sur lui, l'engloutir et s'y méprendre. Le même danger ne guetterait-il pas la pensée de Nietzsche?...

\section{NOTES}

1. - K, IX, 190 (le sigle « $\mathrm{K}$ » désignera l'édition Kröner).

2. $-\mathrm{K}, \mathrm{III}, 68$.

3. - K, II, 420-424.

4. - K, XIV-1, $\$ 400$.

5. $-\mathrm{K}, \mathrm{XI}-2, \S 221$.

6. $-\mathrm{K}, \mathrm{XIII}, \S 131$.

7. - K, XIII, §93.

8. - K, III, §230.

9. - K, XIII, §786.

10. - K, XV, §412.

11. - K, XIII, §237.

12. - K, XIII, §231.

13. - K, XVI, §747.

14. - K, XVI, $\$ 272$.

15. $-\mathrm{K}, \mathrm{I}, 98$.

16. $-\mathrm{K}, \mathrm{I}, 115$.

17. $-\mathrm{K}, \mathrm{I}, 120-125$.

18. - K, I, 224 sq.

19. - K, III-1, 213.

20. - K, II, 188.

21. - K, II, 282.

22. - K, II, 345.

23. - K, III-1, 92.

24. - K, III-1, 168.

25. - K, III-1, 302.

26. - K, VII, 13.

27. - K, VIII, 92.

28. - K, VIII, 121.

29. - K, III-2, 124-125.

30. - K, IV, 301. 


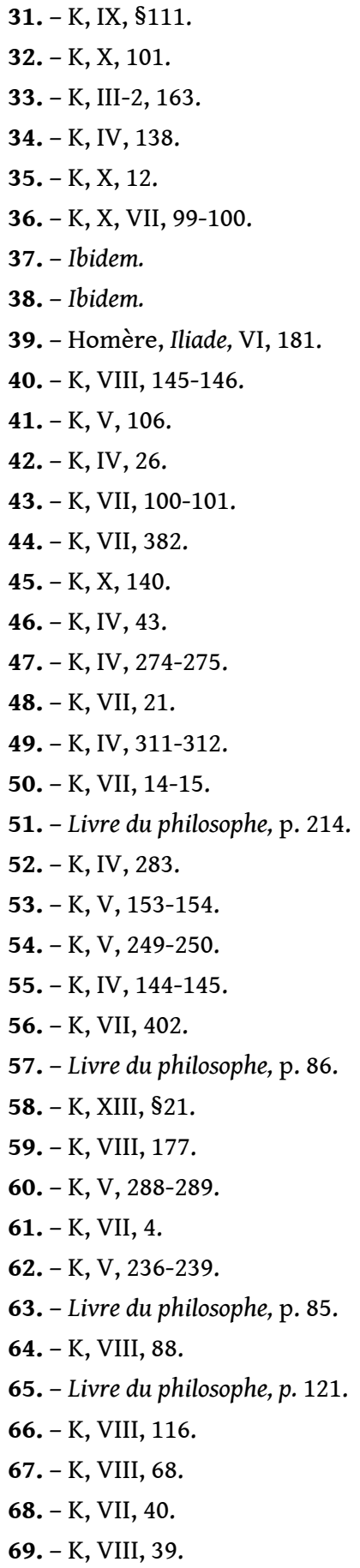

\section{RÉSUMÉS}

L'antiplatonisme de Nietzsche ne doit pas faire illusion. Il y a aussi une attirance, une parenté des deux philosophies. Nietzsche critique l'idéalisme platonicien : le réel vrai n'est pas le sensible, 
mais l'immobilité éternelle des Idées transcendantes. Mais lorsque ces Idées et le bien moral ne furent plus crus des hommes, tout s'effondra : le monde intelligible, puisqu'il était un délire de l'homme, le monde sensible puisque l'idéalisme métaphysique l'avait rejeté, comme illusion et erreur. Critiquer Platon veut donc dire critiquer l'idéalisme métaphysique, sa logique, sa morale, et lui substituer une pensée de la volonté de puissance qui exprime le dépassement de soi des forces qui constituent le chaos dionysiaque de l'être. Mais cette théorie, qui détermine un aristocratisme est-elle bien à l'abri d'une perversion, puisque l'aristocrate Platon fut bien perverti par le moralisme socratique?

Der Antiplatonismus von Nietzsche soll uns nicht über die Tatsache hinwegtäuschen, dass es eine direkte Beziehung, eine Verwandtschaft gibt zwischen den Philosophien beider Denker.

Nietzsche kritisiert den platonischen Idealismus, der behauptet, die wahre Wirklichkeit sei nicht die sinnliche Welt, sondern die ewige Immobilität der transzendenten Ideen. Wenn aber diese Welt der Ideen und die moralischen Grundsätze keine Glaubwürdigkeit mehr haben für die Menschen, hat kein System mehr einen wirklichen Halt, weder die noetische Welt, da der metaphysische Idealismus sie als Täuschung und Irrtum verwirft, noch die sinnliche Welt, da sie eine den Leidenschaften unterworfene Illusion ist. Plato kritisieren, das bedeutet, die idealistische Metaphysik, ihre Logik und ihre Sittenlehre verwerfen und an deren Stelle den Gedanken vom Willen zur Macht setzen, als Ausdruck der Selbstüberwindung der dionysischchaotischen Lebenskräfte. Ist aber diese aristokratisch zugespitzte Theorie nicht der Gefahr einer Missdeutung ausgesetzt, da ja auch der Aristokrat Plato durch den sokratischen Moralismus «verdorben» wurde?

\section{AUTEUR}

\section{PIERRE TROTIGNON}

Université de Lille III 